Anaesthesist 2014 · 63:906-907

DOI 10.1007/s00101-014-2403-y

Online publiziert: 12. November 2014

๑) Springer-Verlag Berlin Heidelberg 2014

\section{F. Wappler}

Klinik für Anästhesiologie und operative Intensivmedizin, Krankenhaus Köln-Merheim, Klinikum der Universität Witten/Herdecke - Köln

\section{Alle Jahre wieder ...}

... erscheint ein Übersichtsbeitrag zum Thema "maligne Hyperthermie“ in den anästhesiologischen Fachzeitschriften. Spricht man Anästhesisten jedoch auf diese Komplikation an, lautet die Antwort zumeist: „Maligne Hyperthermie? Das habe ich noch nie gesehen!" Und es stimmt, die maligne Hyperthermie oder kurz MH ist eine sehr seltene Komplikation, sodass manche sie während ihres gesamten Berufslebens nicht zu Gesicht bekommen. Ist es also wirklich wichtig, über diese Thematik zu berichten? Aber selbstverständlich! Täglich werden weltweit Trigger-Substanzen wie volatile Inhalationsanästhetika und/oder Succinylcholin hunderttausendfach zur Anästhesie von Patienten verwendet. Schwerwiegende Komplikationen sind somit nicht nur möglich, sondern treten naturgemäß auch heute immer wieder auf $[1,2]$.

Daher ist es für alle in der Anästhesie Tätigen, ärztliches und auch pflegerisches Personal, von großer Bedeutung, die Erkrankung, deren klinische Präsentation und die Standards der Therapie genau zu kennen $[3,4]$. Im vorliegenden Übersichtsbeitrag wird zudem auf 2 aktuelle Themen hingewiesen, die zukünftig zu einer Veränderung der Therapie der $\mathrm{MH}$ führen könnten [5].

\section{„Wundermittel“ Dantrolen?}

Vor einigen Jahren wurde in den USA ein neues Dantrolenpräparat entwickelt. Die Besonderheit dieser Substanz besteht in seinen spezifischen pharmakologischen Eigenschaften. Die Substanz mit dem Namen Ryanodex ${ }^{\oplus}$ ist eine nanokristalline Suspension von Dantrolennatrium, deren Applikationsform 150-fach stärker konzentriert ist als das derzeit im Handel verfügbare Dantrolen. Aufgrund der erheblich verbesserten Wasserlöslichkeit des neuen Produkts lassen sich 250 mg Dantrolenwirkstoff in nur $5 \mathrm{ml}$ Wasser vollständig lösen. Die gleiche Dosis des herkömmlichen Wirkstoffs würde hingegen $750 \mathrm{ml}$ Lösungsmittel benötigen. Darüber hinaus lässt sich Ryanodex ${ }^{\oplus}$ deutlich schneller auflösen. In tierexperimentellen Studien wurden die Zeiten bis zum Auflösen der Substanz sowie die Injektionszeiten mit dem Standardpräparat verglichen, und beide Zeiten waren nicht nur statistisch signifikant, sondern auch klinisch relevant verkürzt [6].

Am 23.07.2014 hat nun die Food and Drug Administration Ryanodex ${ }^{\circ}$ ohne umfangreiche Therapiestudien beim Menschen zur Therapie der MH in den USA zugelassen. Die Behörde war offensichtlich davon überzeugt, dass die Subs$\operatorname{tanz}$ eine Optimierung der Behandlung und somit auch der Patientensicherheit ermöglicht. Aufgrund der deutlich verbesserten pharmakologischen Kenndaten des Präparats wäre eine baldige Zulassung in Deutschland ebenfalls wünschenswert. Ob und wann diese erteilt wird, ist jedoch gegenwärtig unklar.

Für die Anästhesie bei einem Patienten mit MH-Disposition wird u. a. die Verwendung eines "dekontaminierten“ Narkosegeräts gefordert. Hierfür wird empfohlen, sämtliche Geräteteile, die mit dem Gasstrom in Verbindung stehen, auszutauschen und das Narkosegerät für $10 \mathrm{~min}$ mit einem Frischgasfluss von 10 1/min zu spülen. Ziel ist die vollständige Eliminierung der Narkosegase aus dem Gerät.

In den vergangenen Jahren wurden jedoch zahlreiche Studien publiziert, die dieses Prozedere infrage stellen [7]. In diesen Untersuchungen wurde an modernen Anästhesiegeräten mit den handelsüblichen Inhalationsanästhetika geprüft, wie lange es bei unterschiedlichen Maßnahmen (Steigerung des Frischgas- flusses, Einsatz eines Aktivkohlefilters etc.) braucht, bis die Konzentration des Gases im Exspirationsschenkel auf 5 ppm reduziert ist. Hierbei konnte nachgewiesen werden, dass Zeiten bis zur Erlangung dieses Zielwerts je nach Auswaschmethode bis maximal $104 \mathrm{~min}$ betragen können. Diese Ergebnisse haben zu einiger Verunsicherung geführt.

\section{Wie soll man also verfahren?}

Die Malignant Hyperthermia Association of the United States empfiehlt, zusätzlich zur klassischen Auswaschprozedur einen Aktivkohlefilter in das System einzubauen, um eine schnelle Dekontamination zu gewährleisten. Alternativ kann jedoch ein frisch aufbereitetes Gerät eingesetzt werden, da nur hierdurch sichergestellt werden kann, dass das Narkosegas komplett eliminiert ist [8]. Mit beiden Konzepten ist man auf der "sicheren Seite", wünschenswert wäre jedoch auch eine klärende Stellungnahme der anästhesiologischen Fachgesellschaften.

Ein relevantes Problem bleibt jedoch: Die MH ist eine sehr seltene Komplikation. Wenn sie eintritt, muss jedoch konsequent, zielgerichtet und korrekt gehandelt werden, um schwerwiegende Folgen für den Patienten zu vermeiden. Dass eine fehlerhafte Therapie deletär sein kann, belegen aktuelle Daten aus den USA. Fünf von 10 Kindern, die eine tödlich verlaufende MH erlitten, hatten kein Dantrolen erhalten [2]. Die Gründe hierfür sind im Einzelnen zwar unklar, aber wichtig ist es, das Anästhesiepersonal nicht nur theoretisch, sondern auch praktisch zu schulen, um beste medizinische Qualität zu bieten. Einen wesentlichen Beitrag zur Problemlösung könnte das „crisis resource management" oder Zwischenfallmanagement im Narkosesimulator liefern [9]. Es 
ist mittlerweile anerkannte Tatsache, dass durch regelmäßiges Training die Qualität der medizinischen Versorgung signifikant verbessert werden kann. Dies gilt insbesondere für selten auftretende Probleme, die einen routinierten Umgang aus der klinischen Tätigkeit allein unmöglich machen. So zeigen erste Studien positive Effekte des Simulatortrainings auch für die $\mathrm{MH}$ [10]. Um für den Krisenfall gerüstet zu sein, ist daher neben der kontinuierlichen theoretischen Fortbildung ein regelmäßiges Training des Anästhesieteams ein wichtiger Beitrag für eine hohe Patientensicherheit.

\section{F. Wayk}

F. Wappler

\section{Korrespondenzadresse}

\section{Prof. Dr. F. Wappler}

Klinik für Anästhesiologie und operative Intensivmedizin, Krankenhaus Köln-Merheim, Klinikum der Universität Witten/Herdecke - Köln Ostmerheimer Str. 200, 51109 Köln

wapplerf@kliniken-koeln.de

\section{Einhaltung ethischer Richtlinien}

Interessenkonflikt. F. Wappler gibt an, dass kein Interessenkonflikt besteht.

\section{Literatur}

1. Larach MG, Brandom BW, Allen GC et al (2014) Malignant hyperthermia deaths related to inadequate temperature monitoring, 2007-2012: a report from the North American Malignant Hyperthermia Registry of the Malignant Hyperthermia Association of the United States. Anesth Analg. DOI 10.1213/ANE.0000000000000421

2. Nelson P, Litman RS (2014) Malignant hyperthermia in children: an analysis of the North American Malignant Hyperthermia Registry. Anesth Analg 118:369-374

3. Roewer N, Schuster F, Wappler F (2008) Empfehlung zur Therapie der malignen Hyperthermie. Anaesth Intensivmed 49:483-488

4. Glahn KP, Ellis FR, Halsall PJ et al (2010) Managing a malignant hyperthermia crisis: guidelines from the European Malignant Hyperthermia Group. Br J Anaesth 105:417-420

5. Metterlein T, Schuster F, Graf BM, Anetseder M (2014) Maligne Hyperthermie. Anaesthesist DOI 10.1007/ s00101-014-2392-x
6. Schütte JK, Becker S, Burmester S et al (2011) Comparison of the therapeutic effectiveness of a dantrolene sodium solution and a novel nanocrystalline suspension of dantrolene sodium in malignant hyperthermia normal and susceptible pigs. Eur J Anaesthesiol 28:256-264

7. Kim TW, Nemergut ME (2011) Preparation of modern anesthesia workstations for malignant hyperthermia-susceptible patients. Anesthesiology 114:205-212

8. Wappler F (2011) Preparation of modern anesthesia workstations for malignant hyperthermiasusceptible patients - when are they really clean? Anesthesiology 115:434-435

9. Østergaard D, Dieckmann P, Lippert A (2011) Simulation and CRM. Best Pract Res Clin Anaesthesiol 25:239-249

10. i Gardi T, Christensen UC, Jacobsen J et al (2001) How do anaesthesiologists treat malignant hyperthermia in a full-scale anaesthesia simulator? Acta Anaesthesiol Scand 45:1032-1035

\section{Ausschreibung: AGNN Preis für Notfallmedizin}

Die Arbeitsgemeinschaft in Norddeutschland tätiger Notärzte e.V. (AGNN) verleiht alle zwei Jahre den von ihr gestifteten „AGNN-Preis für Notfallmedizin“ für herausragende Projekte und / oder wissenschaftliche Arbeiten auf dem Gebiet der Notfallmedizin.

Die Bewerbung erfolgt durch AbstractEinreichung zum Notfallmedzinischen Symposium der AGNN in Travemünde (NOSTRA, 23.-25. April 2015).

An der Auswahl können unveröffentlichte sowie in den beiden Vorjahren der Preisverleihung veröffentlichte Projekte und Arbeiten teilnehmen, die bis zu dem in der jeweiligen Ausschreibung angegebenen Termin bei der AGNN eingereicht werden.

Die angenommenen Arbeiten werden im Rahmen des Notfallsymposiums der AGNN in Travemünde (NOSTRA) in Form einer Posterpräsentation vorgestellt. Die drei bestplatzierten Arbeiten werden im Rahmen des Symposiums als Kurzvortrag (5 Minuten Redezeit und 3 Minuten Diskussion) im Auditorium vorgestellt.

Die Erstautoren dieser Arbeiten werden zur kostenlosen Teilnahme am Symposium eingeladen.

Das Preisgeld beträgt $500,-€$.

Die Statuten sowie das Bewerbungsformular finden Sie unter:

http://www.nostra2015.de/agnn-preis

Quelle: AGNN 